\title{
Effects of Type 2 Diabetes on Brain Structure and Cognitive Function: African American-Diabetes Heart Study MIND
}

\author{
C.T. Whitlow, K.M. Sink, J. Divers, S.C. Smith, J. Xu, N.D. Palmer, C.E. Hugenschmidt, J.D. Williamson, D.W. Bowden,
}

B.I. Freedman, and J.A. Maldjian

o- $\equiv$

\begin{abstract}
BACKGROUND AND PURPOSE: Rates of type 2 diabetes are higher among African Americans compared with individuals of European ancestry. The purpose of this investigation was to determine the relationship between MR imaging measures of brain structure (volume of GM, WM, WM lesions) and cognitive function in a population of African Americans with type 2 diabetes. These MR imaging measures of brain structure are affected by type 2 diabetes-associated macrovascular and microvascular disease and may be associated with performance on tasks of cognitive function in the understudied African American population.
\end{abstract}

MATERIALS AND METHODS: African Americans with type 2 diabetes enrolled in the African American-Diabetes Heart Study MIND study $(n=$ 263) were evaluated across a broad range of cognitive domains and imaged with brain MR imaging. Associations between cognitive parameters and MR imaging measures of whole-brain GM, WM, and WM lesion volumes were assessed by using adjusted multivariate models.

RESULTS: Lower GM volume was associated with poorer performance on measures of general cognitive function, working memory, and executive function. Higher WM lesion volume was associated with poorer performance on a smaller subset of cognitive domains compared with GM volume but included aspects of working memory and executive function. There were no statistically significant associations with WM volume.

CONCLUSIONS: Markers of cortical atrophy and WM lesion volume are associated with cognitive function in African Americans with type 2 diabetes. These associations are described in an African American cohort with disease control similar to that of individuals of European ancestry, rather than underserved African Americans with poor access to health care. Interventions to reduce cortical atrophy and WM disease may improve cognitive outcomes in this understudied population.

ABBREVIATIONS: T2D = type 2 diabetes; HbAlc = hemoglobin Alc; MMSE = Mini-Mental State Examination; MoCA = Montreal Cognitive Assessment; DSC = Digit Symbol Coding Task; VFA = verbal fluency for animals

T he number of individuals with type 2 diabetes (T2D) is increasing throughout the world, and the prevalence of this disease is projected to rise during the next several decades. Estimates

Received October 6, 2014; accepted after revision February 7, 2015.

From the Department of Radiology, Section of Neuroradiology (C.T.W., J.A.M.); Advanced Neuroscience Imaging Research Laboratory (C.T.W., J.A.M.); Department of Biomedical Engineering (C.T.W., J.A.M.); Department of Internal Medicine, Section on Gerontology and Geriatric Medicine (K.M.S., C.E.H., J.D.W.); Division of Pub lic Health Sciences, Department of Biostatistical Sciences (J.D.); Department of Biochemistry (S.C.S., J.X., N.D.P., D.W.B.); Center for Diabetes Research, Center for Genomics and Personalized Medicine Research (N.D.P., D.W.B., B.I.F.); Department of Internal Medicine, Section on Nephrology (B.I.F.), Wake Forest School of Medicine, Winston-Salem, North Carolina.

This work was supported by R01 NS075107 (J.D., J.A.M., B.I.F.), NS058700 (D.W.B.), and DK071891 (B.I.F.).

Please address correspondence to Christopher T. Whitlow, MD, PhD, MHA, Advanced Neuroscience Imaging Research Laboratory, Department of Radiology, Neuroradiology, Translational Science Institute, Wake Forest University Health Sciences, Winston-Salem, NC 27157-1088; e-mail: cwhitlow@wakehealth.edu

-* Indicates open access to non-subscribers at www.ajnr.org

EIndicates article with supplemental on-line table. indicate that diabetes will affect 366 million people in 2030, compared with 171 million in $2000 .{ }^{1} \mathrm{~T} 2 \mathrm{D}$ results in microvascular and macrovascular disease that produces end-organ damage leading to high morbidity and mortality. ${ }^{2}$ Microvascular damage within the brain is associated with cerebral atrophy and other changes thought to underlie T2D-associated cognitive impairment. ${ }^{3-6}$ Furthermore, evidence is growing that the central nervous system effects of T2D may accelerate the rate of cognitive decline among the elderly and increase the risk of developing dementia and Alzheimer disease. ${ }^{7-9}$

Rates of T2D are higher among African Americans compared with individuals of European ancestry. ${ }^{10}$ As such, the prevalence of T2D-associated central nervous system and cognitive sequelae among African Americans may also be higher. Few studies, however, have characterized the association between brain structure and cognitive function in a large comprehensively phenotyped 
cohort of African Americans with T2D, particularly those with relatively well-controlled vascular disease risk factors (hypertension and hyperlipidemia) and access to adequate health care. ${ }^{11}$ The purpose of this investigation was to determine the relationship between MR imaging measures of brain structure and cognitive function in an African American population with T2D enrolled in the African American-Diabetes Heart Study MIND. We hypothesized that lower brain volume and a higher burden of WM disease would be associated with poorer performance on tests spanning a variety of cognitive domains.

\section{MATERIALS AND METHODS \\ Participants}

The Wake Forest University Health Sciences institutional review board approved this study, and all participants provided written informed consent. A cohort of 263 unrelated African Americans with T2D were recruited for this investigation as part of the African American-Diabetes Heart Study MIND, funded by the National Institutes of Health. The objectives of the African American-Diabetes Heart Study MIND are to improve our understanding of the risk factors for impaired cognitive function and abnormalities of cerebral architecture in the understudied African American population with T2D.

Participants included in this study were diagnosed with T2D in the absence of diabetic ketoacidosis with the onset of clinical disease after 30 years of age on the basis of the following: 1) active medical treatment (insulin and/or oral hypoglycemic agents), 2) nonfasting blood sugar of $\geq 200 \mathrm{mg} / \mathrm{dL}, 3$ ) fasting blood sugar of $\geq 126 \mathrm{mg} / \mathrm{dL}$, or 4 ) hemoglobin Alc (HbAlc) of $\geq 6.5 \%$. Fasting measures of glucose, HbAlc, low-density lipoprotein cholesterol, high-density lipoprotein cholesterol, triglycerides, thyroid stimulating hormone, and vitamin $\mathrm{B}_{12}$ were acquired. Medical and educational histories, vital signs, and active medications were also recorded.

\section{Cognitive Testing}

The cognitive battery was organized to evaluate a broad range of cognitive domains known to be associated with brain microvascular disease, which can be present in the setting of T2D. ${ }^{12,13} \mathrm{~A}$ single investigator (K.M.S.) was responsible for quality control and training, certification, and assessment of study staff responsible for conducting cognitive tests. General cognitive function was assessed with the modified Mini-Mental State Examination (MMSE) and Montreal Cognitive Assessment (MoCA). ${ }^{14-16}$ We included the MoCA in addition to the modified MMSE due to its added measures of executive function and increased sensitivity for detecting early cognitive impairment. The Rey Auditory Verbal Learning Test is a word list recall task that evaluates verbal learning and memory ${ }^{17}$ and has been reported to correlate strongly with executive function. ${ }^{18}$ The Digit Symbol Coding Task (DSC) is a subtest of the Wechsler Adult Intelligence Scale (3rd ed) that is used to assess visual motor speed, sustained attention, and working memory, ${ }^{19}$ which have been associated with future cognitive decline. ${ }^{20,21}$ Additional aspects of executive function were evaluated with the verbal fluency for animals (VFA) task and the Stroop test. $^{22-26}$ Depression and anxiety represent possible confounding variables that may affect the relationship between cognitive func- tion and brain structure. Depression and anxiety were evaluated with the Center for Epidemiologic Studies Depression scale ${ }^{27}$ and the Anxiety Brief Symptom Inventory, ${ }^{28}$ respectively.

\section{Brain MR Imaging}

MR Imaging Acquisition. Scanning for the initial 73 subjects was performed on a 1.5T Excite HD scanner (GE Healthcare, Milwaukee, Wisconsin) with twin-speed gradients by using a neurovascular head coil (GE Healthcare). High-resolution T1 anatomic images were obtained by using a 3D volumetric inversion recovery echo-spoiled gradient-echo sequence $(\mathrm{TR}=7.36 \mathrm{~ms}$, $\mathrm{TE}=$ $2.02 \mathrm{~ms}$, TI $=600 \mathrm{~ms}$, flip angle $=20^{\circ}, 124$ sections, FOV $=24$ $\mathrm{cm}$, matrix size $=256 \times 256,1.5-\mathrm{mm}$ section thickness). FLAIR images were acquired in the axial plane $(\mathrm{TR}=8002 \mathrm{~ms}$, $\mathrm{TE}=$ $101.29 \mathrm{~ms}, \mathrm{TI}=2000 \mathrm{~ms}$, flip angle $=90^{\circ}, \mathrm{FOV}=24 \mathrm{~cm}$, matrix size $=256 \times 256,3-\mathrm{mm}$ section thickness). Because of a change in MR imaging equipment at the Wake Forest University Health Sciences Center for Biomolecular Imaging, scanning of the subsequent 190 participants was performed on a 3T Magnetom Skyra MR imaging scanner (Siemens, Erlangen, Germany) by using a high-resolution 20-channel head/neck coil (Siemens). T1weighted anatomic images were obtained by using a 3D volumetric MPRAGE sequence $(\mathrm{TR}=2300 \mathrm{~ms}, \mathrm{TE}=2.99 \mathrm{~ms}, \mathrm{TI}=900$ ms, flip angle $=9^{\circ}, 192$ sections, voxel dimension $=0.97 \times$ $0.97 \times 1 \mathrm{~mm})$. FLAIR images were acquired by using a 3D sampling perfection with application-optimized contrasts by using a different flip angle evolution (Siemens) inversion recovery sequence $(\mathrm{TR}=6000 \mathrm{~ms}, \mathrm{TE}=283 \mathrm{~ms}$, $\mathrm{TI}=2200 \mathrm{~ms}$, flip angle $=$ $120^{\circ}, 160$ sections, voxel dimensions $=1.1 \times 1.1 \times 1 \mathrm{~mm}$ ).

Image Segmentation. Structural T1 images were segmented into GM, WM, and CSF and normalized to Montreal Neurological Imaging space by using the Diffeomorphic Anatomical Registration Through Exponentiated Lie Algebra highdimensional warping (http://www.neurometrika.org/node/ 34) and the SPM8 software (http://www.fil.ion.ucl.ac.uk/spm/ software/spm8 $)^{29}$ new-segment procedure, as implemented in the VBM8 toolbox (http://dbm.neuro.uni-jena.de/vbm.html). Total GM and WM volume and intracranial volume (comprising GM + WM + CSF) were determined from the VBM8 automated segmentation procedure, which outputs a text file with values for native space total GM, WM, and CSF volumes. Whole-brain volumes (GM and WM) are reported milliliters. GM and WM volumes adjusted for intracranial volume were used in the linear regression models.

White Matter Lesion Segmentation. White matter lesion segmentation was performed by using the Lesion Segmentation Toolbox (http://www.academia.edu/2729347/LST_A_Lesion_ Segmentation_Tool_For_SPM_) ${ }^{30}$ for SPM8 at a threshold (k) of $0.25{ }^{31}$ Normalization to Montreal Neurological Imaging space was accomplished by coregistration with the structural T1 and applying the normalization parameters computed in the VBM8 segmentation procedure. The white matter lesion was determined by summing the binary lesion maps and multiplying by the voxel volume; it was reported in milliliters.

AJNR Am J Neuroradiol 36:1648-53 Sep 2015 www.ajnr.org 
Table 1: Demographic and laboratory results in African

American-Diabetes Heart Study MIND participants $(N=263)$

\begin{tabular}{lrr}
\hline \multicolumn{1}{c}{ Variable } & Mean & SD \\
\hline Age (yr) & 60.4 & 9.6 \\
Duration of diabetes (yr) & 14.3 & 8.9 \\
Age of diabetes diagnosis (yr) & 46.0 & 11.0 \\
Body mass index (kg/m²) & 34.1 & 7.9 \\
Glucose (mg/dL) & 147.5 & 60.3 \\
HbAlc (\%) & 8.2 & 2.1 \\
LDL cholesterol (mg/dL) & 109.1 & 37.7 \\
HDL cholesterol (mg/dL) & 47.2 & 11.9 \\
Triglycerides (mg/dL) & 115.7 & 72.3 \\
Systolic blood pressure (mm Hg) & 131.2 & 18.6 \\
Diastolic blood pressure (mm Hg) & 75.4 & 10.8 \\
Thyroid stimulating hormone $(\mu \mathrm{lU} / \mathrm{mL})$ & 2.0 & 1.6 \\
Vitamin B $_{12}(\mathrm{pg} / \mathrm{mL})$ & 690.0 & 417.3 \\
\hline
\end{tabular}

Note:-LDL indicates low-density lipoprotein; HDL, high-density lipoprotein.

\section{Statistical Analyses}

Interscanner Variability. Fifteen study participants underwent scanning by using both $1.5 \mathrm{~T}$ and $3 \mathrm{~T} \mathrm{MR}$ imaging to account for between-scanner variation. Both datasets for each participant were processed by using identical methods. Adjustments were made to account for any systematic differences in volumetric measures between scanners. ${ }^{32,33}$ Linear regression was used to estimate calibration equations between the $1.5 \mathrm{~T}$ and $3 \mathrm{~T}$ measurements. These equations were used to determine the corresponding 3T MR imaging value from an MR imaging scan performed on a $1.5 \mathrm{~T}$ scanner. $R^{2}$ values obtained from the calibration equations also provide an estimate of the reliability of $1.5 \mathrm{~T}$ measurements as predictors of the 3T MR imaging values.

Linear models were fitted to test for associations between measures of cognitive performance (independent variables) and brain MR imaging measures (dependent variables). GM and WM volumes were adjusted for intracranial volume (comprising GM + $\mathrm{WM}+\mathrm{CSF}$ ). The Box-Cox method ${ }^{33}$ was applied to identify the appropriate transformation best approximating the distributional assumptions of conditional normality and the homogeneity of variance of the residuals. This method suggested taking the natural logarithm of WM lesion volume and the squared value of the intracranial volume-adjusted WM and GM volumes. Models were run unadjusted and successively adjusted for age, sex, body mass index, T2D severity (HbA1c), and level of education ( $1=$ less than high school, 2-5 = number of years in high school, $5=$ graduate, $6-9$ number of years in college [ 9 = graduate $], 10=$ postgraduate degree). Adjusted results refer to the model testing for the association between MR imaging variables and cognitive function after adjustment for all 5 covariates. An $\alpha$ level of $\leq .05$ was used for all statistical tests.

\section{RESULTS}

Tables 1 and 2 present demographic and laboratory characteristics of the cohort, and Table 3 contains results of brain MR imaging and cognitive function measurements. The reliability coefficient between MR imaging measures obtained on the 1.5T and 3T scanners ranged between $98 \%$ for the intracranial volume and 92\% for the GM volume. Unadjusted and adjusted analyses were performed to determine the relationships between continuous measures of cerebral anatomy and cognitive function (On-line
Table 2: Group characteristics in African American-Diabetes Heart Study MIND ( $N=263)$

\begin{tabular}{lc}
\hline \multicolumn{1}{c}{ Variable } & No. (\% of Total Cohort) \\
\hline Female & $165(62.7 \%)$ \\
Hypertension & $87(33.1 \%)$ \\
Insulin use & $108(41.1 \%)$ \\
Lipid medication & $93(54.7 \%)$ \\
Smoking & \\
Never & $117(44.7 \%)$ \\
Former & $91(34.7 \%)$ \\
Current & $54(20.6 \%)$ \\
Education & $27(10.3 \%)$ \\
Less than a high school diploma & $76(28.9 \%)$ \\
High school diploma & $115(43.7 \%)$ \\
Some college or a college diploma & $45(17.1 \%)$ \\
Graduate education &
\end{tabular}

Table 3: MRI and cognitive function measures in African American-Diabetes Heart Study MIND

\begin{tabular}{lrrr}
\multicolumn{1}{c}{ Variable } & Mean & SD & No. \\
\hline MRI results & & & \\
GM volume, unadjusted (mL) & 732.2 & 57.7 & 263 \\
GM/ICV (\%) & 43.3 & 2.8 & 263 \\
WM volume, unadjusted (mL) & 629.6 & 46.7 & 263 \\
WM/ICV (\%) & 37.3 & 2.3 & 263 \\
WM lesion volume (mL) & 7.9 & 14.7 & 263 \\
Cognitive function measures & & & \\
3MSE (score, 0-100) & 84.6 & 9.0 & 256 \\
MoCA (score, 0-30) & 19.9 & 3.9 & 261 \\
RAVLT (score, 0-75) & 38.0 & 9.0 & 263 \\
DSC (score, 0-133) & 48.1 & 15.9 & 262 \\
VFA & 15.7 & 4.6 & 262 \\
Stroop errors & 3.4 & 6.3 & 258 \\
Stroop interference (seconds; trial 3 to trial 2) & 31.6 & 16.1 & 256 \\
CESD & 8.0 & 5.3 & 262 \\
BSI-Anxiety & 4.9 & 4.5 & 262 \\
\hline
\end{tabular}

Note:-ICV indicates intracranial volume; 3 MSE, modified MMSE; RAVLT, Rey Auditory Verbal Learning Test; CESD, Center for Epidemiologic Studies Depression Scale; BSI-Anxiety, Brief Symptom Inventory.

a Whole-brain measures adjusted for total ICV.

Table). Results of the fully adjusted models, including the effects of age, sex, body mass index, HbAlc, and level of education, follow. No significant relationships were detected between glycemic control assessed by $\mathrm{HbA1c}$ with WM lesion volume, GM volume, or WM volume $(P=.51, P=.36$, and $P=.35$, respectively; data not shown). There were also no statistically significant associations between MR imaging measures and the depression and anxiety evaluations $(P>.05)$.

As shown in the On-line Table, the modified MMSE had a positive association with GM volume $(P=.0023)$ but no statistically significant association with WM volume $(P=.18)$ or WM lesion volume $(P=.15)$. The MoCA had a positive association with GM volume $(P=.022)$, but no significant association with WM volume $(P=.43)$ or WM lesion volume $(P=.82)$. DSC had a positive association with GM volume $(P=.015)$ and a negative association with WM lesion volume $\left(P=6.7 \times 10^{-4}\right)$, but no significant association with WM volume $(P=.12)$. Stroop interference had a negative association with GM volume $(P=.035)$ and a positive association with WM lesion volume $(P=4.6 \times$ $\left.10^{-3}\right)$, but no significant association with WM volume $(P=.26)$. The Rey Auditory Verbal Learning Test, VFA, and Stroop errors were not significantly associated with any of the brain MR imag- 
ing measures $(P>.05)$. Measures of effect size are provided in the last columns of the On-line Table for lower GM and WM and higher WM lesion volume associated with cognitive test performance. The subcolumns labeled "Absolute" and "\%" report the absolute and percentage differences from the mean cognitive score computed for each $1 \mathrm{SD}$ change in MR imaging measure. For example, $1 \mathrm{SD}$ lower GM volume corresponds to lower modified MMSE ("Absolute," “\%”: $-10.7,-12.6 \%)$, MoCA (-1.2, $-6.2 \%)$, and DSC $(-2.7,-5.5 \%)$ scores and a higher Stroop interference $(2.1,6.8 \%)$ score compared with group mean scores reported in Table 3. A 1-SD higher WM lesion volume corresponds to lower DSC ("Absolute," “\%”: $-3.3,-6.9 \%$ ) and higher Stroop interference $(2.9,9.0 \%)$ scores compared with group mean scores reported in Table 3.

\section{DISCUSSION}

The African American-Diabetes Heart Study MIND was designed to improve our understanding of T2D-associated risk factors for impaired cognitive function and abnormalities of cerebral architecture in the understudied African American population with levels of disease control similar to those of individuals of European ancestry. In general, lower whole-brain GM volume and higher WM lesion volume were associated with poorer cognitive performance in this cohort. Measures of GM atrophy, as characterized by lower GM volume, were associated with the greatest number of cognitive domains evaluated, including a variety of general measures of cognitive function, working memory, and executive function. WM lesion volume was associated with a smaller subset of cognitive domains compared with GM volume, including aspects of working memory and executive function. In contrast to measures of GM atrophy and WM lesion volume, there were no statistically significant associations between any measures of cognitive function and WM volume. Furthermore, a few measures of cognitive function demonstrated no associations with measures of cerebral anatomy. Specifically, there were no statistically significant associations with verbal fluency, as measured with VFA, and any of the brain MR imaging measures.

As opposed to prior studies of brain anatomy and cognition in African Americans with T2D, participants in the African American-Diabetes Heart Study MIND had relatively good access to health care. ${ }^{34,35}$ This is reflected by high rates of statin use and mean blood pressures, universal treatment for hypertension, and $\mathrm{HbAlc}$ and lipid profiles reflecting a level of control seen in studies comprising subjects with European ancestry. As such, the results of this study may not be generalizable to African Americans with T2D that is poorly monitored and/or controlled. T2D is known to be associated with structural changes in the brain, similar to those reported in our study, including cerebral atrophy and WM lesions that correlate with the presence of microvascular and macrovascular ischemic disease. ${ }^{36-41}$ Other cross-sectional studies of T2D have reported associations among brain atrophy, WM lesion burden, and diminished cognitive function. In 1 study, MR imaging variables were used in a stepwise regression model that demonstrated the strongest relationship between GM atrophy and cognitive function, with WM volume and WM lesion volume demonstrating relatively smaller effects on the model. ${ }^{6}$

These findings may have relevance in the context of our data in which GM volume was associated with the greatest number of cognitive domains evaluated and WM lesion volume was associated with a smaller subset of cognitive functions. Such findings regarding brain anatomy and cognitive function are important because it is thought that T2D-associated changes in the brain may underlie the increased risk of developing dementia among this population, including a reported $50 \%-100 \%$ increased risk of developing Alzheimer disease. ${ }^{42-44}$ Very likely many factors link MR imaging measures of cerebral anatomy and cognitive function. However, adjustment for age, sex, body mass index, T2D severity (HbA1c), and level of education did not affect these relationships in this study. These findings suggest that the impact of diabetes on brain volume, WM lesion load, and cognitive function that we report was not directed through these pathways.

Our results provide an extensive characterization of brain structure and cognitive function focused solely on the understudied African American population with T2D and well-controlled cardiovascular disease risk factors. Strengths include the relatively large sample size of African Americans with diabetes and the breadth of cognitive functions evaluated, which included a variety of domains previously shown to be affected by T2D. This study also used quantitative structural MR imaging measures that have been applied in numerous studies of T2D. However, this study adds to the previous literature by using fully quantitative measures of WM lesion volume previously validated in this cohort, ${ }^{31}$ rather than qualitative and semiquantitative measures of WM lesion load.

The limitations of this study include the lack of a nondiabetic control group, cross-sectional design, and lack of covariates that could be related to potential mechanisms, such as medication use and inflammatory markers. This study also focused on wholebrain MR imaging measures rather than evaluating individual brain ROIs such as the hippocampus and frontal lobes, which may more specifically underlie performance in the cognitive domains evaluated. Such investigations of specific ROIs will be conducted in the future once data collection for the entire African AmericanDiabetes Heart Study MIND cohort is completed.

\section{CONCLUSIONS}

Results from the African American-Diabetes Heart Study MIND cohort reveal that cognitive function is associated with markers of cortical atrophy and WM lesion volume, independent of diabetes severity in African Americans with T2D. These associations between brain structure and cognitive function are described in a cohort of African Americans with disease control similar to that of individuals of European ancestry, rather than underserved African Americans with poor access to health care. Taken together, our data suggest that interventions to reduce cortical atrophy and white matter disease may have the potential to improve cognitive outcomes in this understudied population.

\section{ACKNOWLEDGMENTS}

The authors thank the African American-Diabetes Heart Study MIND participants and study staff for their valuable contributions. 
Disclosures: Christopher T. Whitlow-RELATED: Grant: National Institutes of Health, ${ }^{*}$ National Institute of Neurological Disorders and Stroke, ${ }^{\star}$ Comments: R01 NS075107. Kaycee M. Sink—RELATED: Grant: National Institutes of Health (National Institute of Neurological Disorders and Stroke)*; UNRELATED: Grants/Grants Pending: Navidea Pharmaceuticals, ${ }^{*}$ Alzheimer's Association, ${ }^{*}$ Comments: 1) Navidea Pharmaceuticals, site Principal Investigator for a clinical trial involving an amyloid imaging agent, 2) Alzheimer's Association, study clinician on an investigator-initiated grant, 3) all other grant funding is federal or not relevant to this work. Jasmin DiversRELATED: Grant: National Institutes of Health. ${ }^{*}$ Christina E. Hugenschmidt-RELATED: Grant: National Institute of Diabetes and Digestive and Kidney Diseases.* Jeff D. Williamson—RELATED: Grant: National Institutes of Health.* Barry I. FreedmanRELATED: Grant: National Institutes of Health, ${ }^{\star}$ Comments: The African AmericanDiabetes Heart Study MIND is a National Institutes of Health-funded research study (R01). Drs Maldjian, Divers, and Freedman are co-Principal Investigators on the grant. Joseph A. Maldjian—RELATED: Grant: National Institutes of Health,* Comments: R01-funded study through the National Institute of Neurological Disorders and Stroke. *Money paid to the institution.

\section{REFERENCES}

1. Wild S, Roglic G, Green A, et al. Global prevalence of diabetes: estimates for the year 2000 and projections for 2030. Diabetes Care 2004;27:1047-53

2. Simić I, Pecin I, Tedeschi-Reiner E, et al. Risk factors for microvascular atherosclerotic changes in patients with type 2 diabetes mellitus. Coll Antropol 2013;37:783-87

3. Biessels GJ. Cerebral complications of diabetes: clinical findings and pathogenetic mechanisms. Neth J Med 1999;54:35-45

4. van Harten B, de Leeuw FE, Weinstein $\mathrm{HC}$, et al. Brain imaging in patients with diabetes: a systematic review. Diabetes Care 2006;29:2539-48

5. van Elderen SG, de Roos A, de Craen AJ, et al. Progression of brain atrophy and cognitive decline in diabetes mellitus: a 3-year followup. Neurology 2010;75:997-1002

6. Moran $\mathrm{C}$, Phan TG, Chen J, et al. Brain atrophy in type 2 diabetes: regional distribution and influence on cognition. Diabetes Care 2013;36:4036-42

7. Vagelatos NT, Eslick GD. Type 2 diabetes as a risk factor for Alzheimer's disease: the confounders, interactions, and neuropathology associated with this relationship. Epidemiol Rev 2013;35:152-60

8. Ryan JP, Fine DF, Rosano C. Type 2 diabetes and cognitive impairment: contributions from neuroimaging. J Geriatr Psychiatry Neurol 2014;27:47-55

9. Sato N, Morishita R. Roles of vascular and metabolic components in cognitive dysfunction of Alzheimer disease: short- and long-term modification by non-genetic risk factors. Front Aging Neurosci 2013;5:64

10. Peek ME, Cargill A, Huang ES. Diabetes health disparities: a systematic review of health care interventions. Med Care Res Rev 2007;64 (5 suppl):101S-56S

11. Divers J, Hugenschmidt C, Sink KM, et al. Cerebral white matter hyperintensity in African Americans and European Americans with type 2 diabetes. J Stroke Cerebrovasc Dis 2013;22:e46-52

12. Thabit H, Kyaw Tun T, McDermott J, et al. Executive function and diabetes mellitus: a stone left unturned? Curr Diabetes Rev 2012;8:109-15

13. Qiu C, Sigurdsson S, Zhang Q, et al. Diabetes, markers of brain pathology, and cognitive cognition: the age, gene/environment susceptibility-Reykjavik study. Ann Neurol 2014;75:138-46

14. Teng EL, Chui HC. The Modified Mini-Mental State (3MS) Examination. J Clin Psychiatry 1987;48:314-18

15. Grace J, Nadler JD, White DA, et al. Folstein vs modified Mini-Mental State Examination in geriatric stroke: stability, validity, and screening utility. Arch Neurol 1995;52:477-84

16. Nasreddine ZS, Phillips NA, Bedirian V, et al. The Montreal Cognitive Assessment, MoCA: a brief screening tool for mild cognitive impairment. J Am Geriatr Soc 2005;53:695-99

17. Strauss E, Sherman S, Spreen O. A Compendium of Neuropsychological
661 Tests: Administration, Norms, and Commentary. 3rd ed. New York: Oxford University Press; 2006

18. Duff K, Schoenberg MR, Scott JG, et al. The relationship between executive functioning and verbal and visual learning and memory. Arch Clin Neuropsychol 2005;20:111-22

19. Wechsler D. Wechsler Adult Intelligence Scale. 3rd ed. San Antonio: Psychological Corp; 1997

20. Hoyer WJ, Stawski RS, Wasylyshyn C, et al. Adult age and digit symbol substitution performance: a meta-analysis. Psychol Aging 2004;19:211-14

21. Rosano C, Simonsick EM, Harris TB, et al. Association between physical and cognitive function in healthy elderly: the health, aging and body composition study. Neuroepidemiology 2005;24:8-14

22. MacLeod CM. Half a century of research on the Stroop effect: an integrative review. Psychol Bull 1991;109:163-203

23. Houx PJ, Jolles J, Vreeling FW. Stroop interference: aging effects assessed with the Stroop Color-Word Test. Exp Aging Res 1993;19:209-24

24. Klein M, Ponds RW, Houx PJ, et al. Effect of test duration on agerelated differences in Stroop interference. J Clin Exp Neuropsychol 1997; 19:77-82

25. Van der Elst W, Molenberghs G, Van Boxtel MP, et al. Establishing normative data for repeated cognitive assessment: a comparison of different statistical methods. Behav Res Methods 2013;45:1073-86

26. Reis JP, Launer LJ, Terry JG, et al. Subclinical atherosclerotic calcification and cognitive functioning in middle-aged adults: the CARDIA study. Atherosclerosis 2013;231:72-77

27. Radloff LS. The CES-D scale: a self-report depression scale for research in the general population. Appl Psychol Meas 1977;1:385-401

28. Gilbar O, Ben-Zur H. Adult Israeli community norms for the Brief Symptom Inventory (BSI). Int J Stress Manage 2002;9:1-10

29. Ashburner J, Friston KJ. Voxel-based morphometry: the methods. Neuroimage 2000;11:805-21

30. Schmidt P, Gaser C, Arsic M, et al. An automated tool for detection of FLAIR-hyperintense white-matter lesions in multiple sclerosis. Neuroimage 2012;59:3774-83

31. Maldjian JA, Whitlow CT, Saha BN, et al. Automated white matter total lesion volume segmentation in diabetes. AJNR Am J Neuroradiol 2013;34:2265-70

32. Jovicich J, Czanner S, Han X, et al. MRI-derived measurements of human subcortical, ventricular and intracranial brain volumes: reliability effects of scan sessions, acquisition sequences, data analyses, scanner upgrade, scanner vendors and field strengths. Neuroimage 2009;46:177-92

33. Han X, Jovicich J, Salat D, et al. Reliability of MRI-derived measurements of human cerebral cortical thickness: the effects of field strength, scanner upgrade and manufacturer. Neuroimage 2006; 32:180-94

34. Aggarwal NT, Wilson RS, Bienias JL, et al. The association of magnetic resonance imaging measures with cognitive function in a biracial population sample. Arch Neurol 2010;67:475-82

35. Brickman AM, Schupf N, Manly JJ, et al. Brain morphology in older African Americans, Caribbean Hispanics, and whites from northern Manhattan. Arch Neurol 2008;65:1053-61

36. Kumar R, Anstey KJ, Cherbuin N, et al. Association of type 2 diabetes with depression, brain atrophy, and reduced fine motor speed in a 60- to 64-year-old community sample. Am J Geriatr Psychiatry 2008;16:989-98

37. Biessels GJ, Koffeman A, Scheltens P. Diabetes and cognitive impairment: clinical diagnosis and brain imaging in patients attending a memory clinic. J Neurol 2006;253:477-82

38. Schmidt R, Launer LJ, Nilsson LG, et al. Magnetic resonance imaging of the brain in diabetes: the Cardiovascular Determinants of Dementia (CASCADE) study. Diabetes 2004;53:687-92

39. Manschot SM, Biessels GJ, de Valk H, et al. Metabolic and vascular determinants of impaired cognitive performance and abnormali- 
ties on brain magnetic resonance imaging in patients with type 2 diabetes. Diabetologia 2007;50:2388-97

40. Kumar A, Haroon E, Darwin C, et al. Gray matter prefrontal changes in type 2 diabetes detected using MRI. J Magn Reson Imaging 2008;27:14-19

41. de Bresser J, Tiehuis AM, van den Berg E, et al. Progression of cerebral atrophy and white matter hyperintensities in patients with type 2 diabetes. Diabetes Care 2010;33:1309-14
42. Biessels GJ, Staekenborg S, Brunner E, et al. Risk of dementia in diabetes mellitus: a systematic review. Lancet Neurol 2006;5:64-74

43. Biessels GJ, Deary IJ, Ryan CM. Cognition and diabetes: a lifespan perspective. Lancet Neurol 2008;7:184-90

44. Kloppenborg RP, van den Berg E, Kappelle LJ, et al. Diabetes and other vascular risk factors for dementia: which factor matters most? A systematic review. Eur J Pharmacol 2008;585:97-108 